\title{
Editorial
}

\section{Obsessive-compulsive disorder}

Nir Lipsman, M.D., And Andres M. Lozano, M.D., Ph.D.

Division of Neurosurgery, Toronto Western Hospital, University of Toronto, Ontario, Canada

Obsessive-compulsive disorder (OCD) is among the most common anxiety disorders and one of the most challenging psychiatric conditions to treat. Despite several classes of medications and multiple schools of psychotherapy, a significant proportion of patients remain symptomatic and disabled by their illness. There is therefore an important need to examine alternative approaches to management, a need that has been recognized for well over half a century, since the first published reports of surgery for "obsessional neurosis."

In this study, Sheth et al. ${ }^{3}$ review their institution's experience with ablative limbic system surgery in an openlabel trial in 64 consecutive cases of treatment-resistant OCD. They report that all 64 patients underwent cingulotomy, with 30 of those requiring at least one additional procedure: either another cingulotomy or conversion to subcaudate tractotomy. At longest follow-up of more than 5 years, the authors report that $47 \%$ of patients met the criteria for full response (defined as a $\geq 35 \%$ decrease in the Yale-Brown Obsessive Compulsive Scale score), with an additional $22 \%$ of patients reaching partial response criteria (decrease of $\geq 25 \%$ in the Yale-Brown Obsessive Compulsive Scale score). These are encouraging and positive results, especially given the extent of previous treatment failure and the degree of treatment resistance that the authors carefully established in these patients.

Although no treatment can claim a complete response in all patients, this is especially true in OCD, for which both the condition and its treatments are equally diverse and heterogeneous. It is therefore not entirely surprising that just under half of the patients required 2 and sometimes 3 procedures to achieve symptom control. This underscores the importance of close follow-up, judicious assessments, and careful weighing of additional therapeutic options. Additional procedures, however, increase risk, and although the authors do demonstrate the relative safety of their procedure, the occurrence of intraoperative generalized seizures and postoperative abulia in some of their patients is a reminder that limbic surgery is not without its potential pitfalls.
Most published series and trials studying surgery for OCD, be it ablation or stimulation, report relatively similar rates of remission and response. This is a little perplexing given the diverse array of targets, including the anterior limb of the internal capsule, the ventral caudate and striatum, inferior thalamic peduncle, and subthalamic nucleus. Perhaps common underlying circuits are influenced by these various procedures. Independent of target and treatment modality, other critical factors contribute to patient outcomes, including treatment expectations, social support, and possibly genetic interactions.

Inevitably, the results of ablative surgery in OCD will be compared to those in the deep brain stimulation (DBS) literature, with proponents of the latter touting the reversibility and measurability of that procedure, and its suitability for blinded assessments. The authors rightly point out that DBS is a resource-intensive procedure that in its current, largely investigative applications necessitates heavy involvement of a dedicated research team that can unintentionally and indirectly influence patient expectations and outcomes. It is important to note, however, that this is a feature of all major clinical trials, surgical or otherwise, and is not specific to DBS or OCD. Furthermore, it may be that DBS and ablative procedures are not mutually exclusive, and as has been shown in depression, perhaps there is room for both in the treatment algorithm, even within the same patient. ${ }^{2}$

Sheth et al. should be commended not only for their large series and encouraging results, but also for their emphasis on judicious record keeping, careful longitudinal assessments, and individualization of care in patients who remain symptomatic. This may well be the optimal approach for a condition as heterogeneous as OCD, whose treatment remains as complex as ever. (http://thejns.org/doi/abs/10.3171/2012.7.JNS12841)

\section{Disclosure}

Dr. Lozano is a consultant for Medtronic and for St. Jude Medical, has an ownership interest in Boston Scientific, and is a patent holder with Functional Neurosciences, Inc. Dr. Lipsman reports no conflict of interest.

\section{References}

1. Dibden WA: Prefrontal leucotomy for obsessional neurosis: report of a case. Med J Aust 2:511, 1947

2. Neimat JS, Hamani C, Giacobbe P, Merskey H, Kennedy SH, Mayberg HS, et al: Neural stimulation successfully treats de- 
pression in patients with prior ablative cingulotomy. Am J Psychiatry 165:687-693, 2008

3. Sheth SA, Neal J, Tangherlini F, Mian MK, Gentil A, Cosgrove GR, et al: Limbic system surgery for treatment-refractory obsessive-compulsive disorder: a prospective long-term followup of 64 patients. Clinical article. J Neurosurg [epub ahead of print December 14, 2012. DOI: 10.3171/2012.11.JNS12389]

\section{Response}

Sameer A. Sheth, M.D., Ph.D., And Emad N. Eskandar, M.D.

\author{
Department of Neurosurgery, Massachusetts General Hospital, \\ Boston, Massachusetts
}

We thank Drs. Lipsman and Lozano for their thoughtful analysis of our study and its implications. They highlight some interesting points regarding the surgical treatment of refractory OCD. First, we certainly agree that it is somewhat remarkable that modulation (stimulation or ablation) of a variety of different targets produces relatively similar outcomes. These results suggest that these regions (cingulate, anterior limb of internal capsule, inferior thalamic peduncle, and subthalamic nucleus) may be interconnected in a limbic/affective circuit whose dys- function results in the expression of OCD symptoms. We can further hypothesize that different subtypes of OCD, as defined by their respective obsessive and compulsive manifestations, may respond differently to modulation of various nodes in the circuit. As our collective experience accumulates with psychiatric neurosurgical procedures, these nuances will be important to address.

Second, it is essential that candidacy for surgery as well as postoperative care is guided by a multidisciplinary group consisting of psychiatrists, neurologists, and neurosurgeons. These patients must be selected carefully and monitored closely by a team that can recognize all possible demonstrations of neurological or psychiatric deterioration.

Finally, as we learn more about the circuits involved it is certainly possible that some patients may benefit from a combination of DBS and ablative procedures, as Drs. Lipsman and Lozano suggest. Thorough study of the functional anatomy and physiology of these targets must proceed in parallel with clinical trials of the procedures' efficacy to optimize treatment for these patients with refractory OCD.

\footnotetext{
Please include this information when citing this paper: published online December 14, 2012; DOI: 10.3171/2012.7.JNS12841.
} 